TUTA/IOE/PCU

Journal of the Institute of Engineering, Vol. 8, No. 1, pp. 25-38 C TUTA/IOE/PCU

All rights reserved. Printed in Nepal Fax: 977-1-5525830

\title{
Experimental Performance Analysis for 2G/3G Cellular Networks based on Mobile Terminals
}

\author{
M. Jiménez-Licea, S. Vidal-Beltrán, J. López-Bonilla \\ Maestría en Ciencias en Ingeniería de Telecomunicaciones, \\ Instituto Politécnico Nacional, Escuela Superior de Ingeniería Mecánica y Eléctrica, \\ Edif. Z-4, 3er. Piso, Col. Lindavista, CP 07738, México DF, \\ montserratlicea@gmail.com, sergiovidalb@mac.com, jlopezb@ipn.mx
}

\begin{abstract}
This work presents the experimental results related to performance measurements in GSM, EDGE and WCDMA cellular data networks. Two Mobile Terminals were used, in order to take place the scenario. Power Level, Power vs Time, Modulation Analysis, Modulation Spectrum, (BER) Bit Error Rate and Throughput were considered in this analysis.
\end{abstract}

Keywords - component: GSM, EDGE, WCDMA, BER/BLER, Throughput

\section{Introduction}

WCDMA (Wideband Code Division Multiple Access) is a technology of third generation 3G that increases data transmission rates by broadening the signal at the air interface through the Multiple Access Code Division Multiplexing instead of Time Division used by GSM systems. WCDMA supports multimedia services with very high speed and full-motion video, Internet access and videoconferencing. It also easily handles applications that make use intensively of bandwidth such as data and image transmission via Internet. In a communication system based on multiple access, an amount of users want to access the channel simultaneously. To implement a multiple access communication there are several techniques that allow users to share the channel. In the technique of multiple access code division -CDMA-, users can access the channel simultaneously and share the same bandwidth.

WCDMA is a spread spectrum technology [1], which expands the signals over a bandwidth of 5 $\mathrm{MHz}$ and is capable of carrying voice and data at the same time. These features allow a data rate of $384 \mathrm{kbps}$ in Release 5, and a transfer rate in the downlink of up to 2Mbps in the Release 6, which includes HSPA, and average processing speeds (for file downloads) of 220-320 Kbps [2].

Spread spectrum is a technique used to transmit data on a bandwidth considerably larger. Its basic foundation is the "widening" of the transmitted signal over a very wide frequency band, much wider than the minimum bandwidth required transmitting the information that you want to send. This higher bandwidth can be obtained by encoding information with a pseudo-random signal. The coded information is transmitted in the frequency that works for which the sender uses a much higher bandwidth than no encryption is used (direct sequence). The spreading operation is the multiplication of each bit of user data with a sequence of 8 bits of code, called 
chips [3-4]. Currently, WCDMA is the wireless access technology broadest deployed worldwide. WCDMA is a wide band system where the transmitted signal with a rate $\mathrm{R}$ is spread by combining it with a wideband spreading signal, creating a spread signal with a bandwidth $\mathrm{W}$. The effective bandwidth for a WCDMA air interface is $3.84 \mathrm{MHz}$ and with guard bands, as a result the required bandwidth is $5 \mathrm{MHz}$ [4]. The WCDMA system uses several codes; when the signal is transmitted from the Base Station carries a unique code called Scrambling Code SC, used in the downlink direction for cell/sector separation. This SC is also employed in the uplink direction to separate every user from each other.

Cellular networks were created to transmit voice calls, but currently, it is able to carry multimedia services like data and video. In the following section, the cellular communication generations are described.

In the other hand, the mobile terminal is a critical issue inside of a Cellular Communication System, due to its characteristics of transmission and reception; they defined the quality in the data and voice transmission. This work carried out several experiments mainly focused on the following parameters:

- Power Measurements

- Data transmission measurements

- BER and BLER Tests

GSM, GPRS and WCDMA were the technologies selected in order to deploy the performance analysis. This kind of work cab be used for manufacturers, service providers and user in order to verify the capacity and coverage of cellular data networks.

\section{Performance Parameters in Mobile Terminals in GSM System}

The mobile terminal is a critical issue inside of a Cellular Communication System, due to its characteristics of transmission and reception; they defined the quality in the data and voice transmission. In this work, some experiments of the most important parameters in Mobile terminal were carried out. The following figure shows the scenery that is necessary to set up to be able to get the experimental results $[2,3]$.

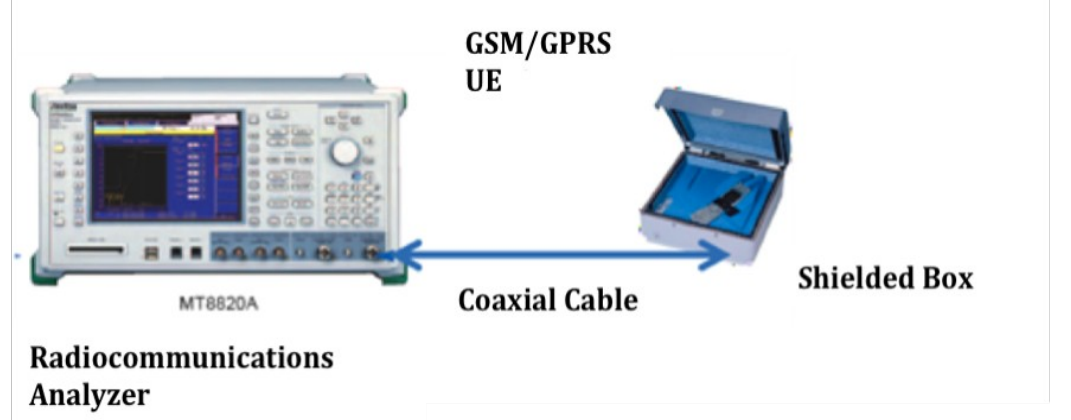

Figure 3: Test Scenario.

\section{Experimental scenario}

The elements that are necessary to figure out the experimental scenery are:

- MT8820A Radiocommunications Analyzer, it has a range of frequencies between 30 $\mathrm{MHz}$ to $2.7 \mathrm{GHz}$, The range result enough to get the experimental measures into the cell 
phones. This equipment allowed getting measurements of Transmission/Reception in technologies like: W-CDMA/HSDPA, GSM/GPRS/EGPRS, cdma2000, 1x (IS-2000), cdma2000 1xEV-DO, PDC and PHS/ADVANCED [2,3].

- Shielded Box is used to avoid interferences from the surrounding Base Stations. Such device works between 800 and $2500 \mathrm{MHz}$, and gives $60 \mathrm{~dB}$ of isolation [2, 3].

- Coaxial cable is used to connect the MT8820A to the Shielded box. Type-N connectors are used.

- Two mobile terminals that operate in GSM, as much as WCDMA; they are iPhone 3G and Nokia 6210.

- A USIM card is installed for the test. It was necessary to register the USIM with the Radiocommunications analyzer.

\section{Power measurements in voice calls}

Tests were made in the Radiocommunications Laboratory of the Master Degree in Telecommunications Engineering (IPN). Once the equipments were properly installed, the following procedure took place [4].

- The Radiocommunications analyzer was powered up, and the technology parameters were configured, according to GSM, GPRS, EDGE or WCDMA technology.

- Voice transmission parameters were configured, in order to establish a voice call.

- The UE is powered up and was placed into the shielded box. The shielded box allows to focus on the power inside the box and avoiding electromagnetic interferences from the surrounding cellular antennas

- The MTT8820A is configured with a power between $-40 \mathrm{dBm}$ to $-50 \mathrm{dBm}$

- UE establish communications with the Analyzer and the state Registered have to appear in the MT8820A's screen.

- The power level was slowly decremented until the Analyzer detects errors

- The Power levels are registered, as much as the errors that the Analyzer captured.

\section{Data Transmission Measurements}

Once the equipment, described in the previous section, was properly installed, the following procedure took place.

- The Radiocommunications analyzer was powered up, and the technology parameters were configured, according to GSM, GPRS, EDGE or WCDMA technology.

- Voice transmission parameters were configured, in order to establish a voice call.

- The UE is powered up and was placed into the shielded box. The shielded box allows to focus on the power inside the box and avoiding electromagnetic interferences from the surrounding cellular antennas

- The MTT8820A is configured with a power between $-40 \mathrm{dBm}$ to $-50 \mathrm{dBm}$

- UE establish communications with the Analyzer and the state Registered have to appear in the MT8820A's screen. 
- A data Server (http) is connected into the same network segment that the MT8820A, and the connectivity between them is verified

- The UE makes a data request in order that to download a file with a size previously defined

- The download times are registered.

\section{Power measurements in voice calls for GSM}

Figure 5, shows the parameters set up of the MT8820A for GSM [5].

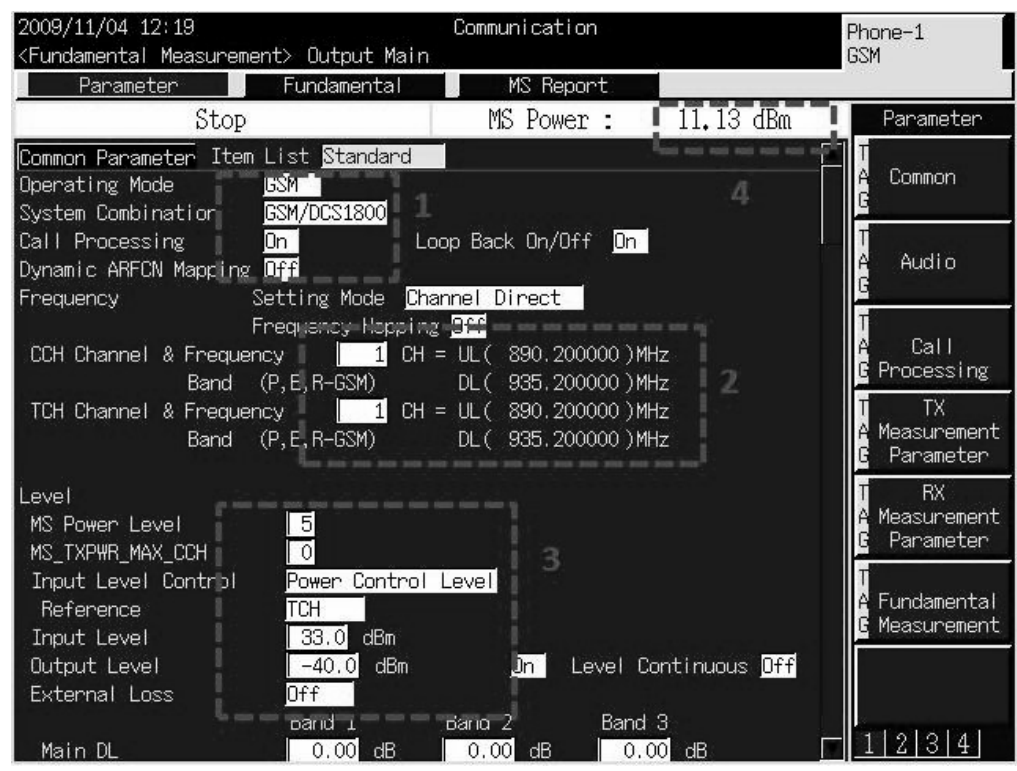

Figure 5.Configuration Parameters in GSM

In the Figure 5, in the first part has showed that GSM is configured, in the part 2, just like the frequency in the channel in the downlink and uplink; The power in the base station is specified in the section $3(-40 \mathrm{dBm})$, the power in the mobile station $(33 \mathrm{dBm})$ and it is used the control of power, the last in the part 4 it is showed the power in the base station that it is received for the mobile station $(11.13 \mathrm{dBm})$.

Initially, the measurement was made on the Nokia 6120, those were: Power Level, Power vs Time, Modulation Analysis, Modulation Spectrum, (BER) Bit Error Rate, the performance in the mobile terminal shows an acceptable level in the range allowed in the specification, the figures 67 show some results. 


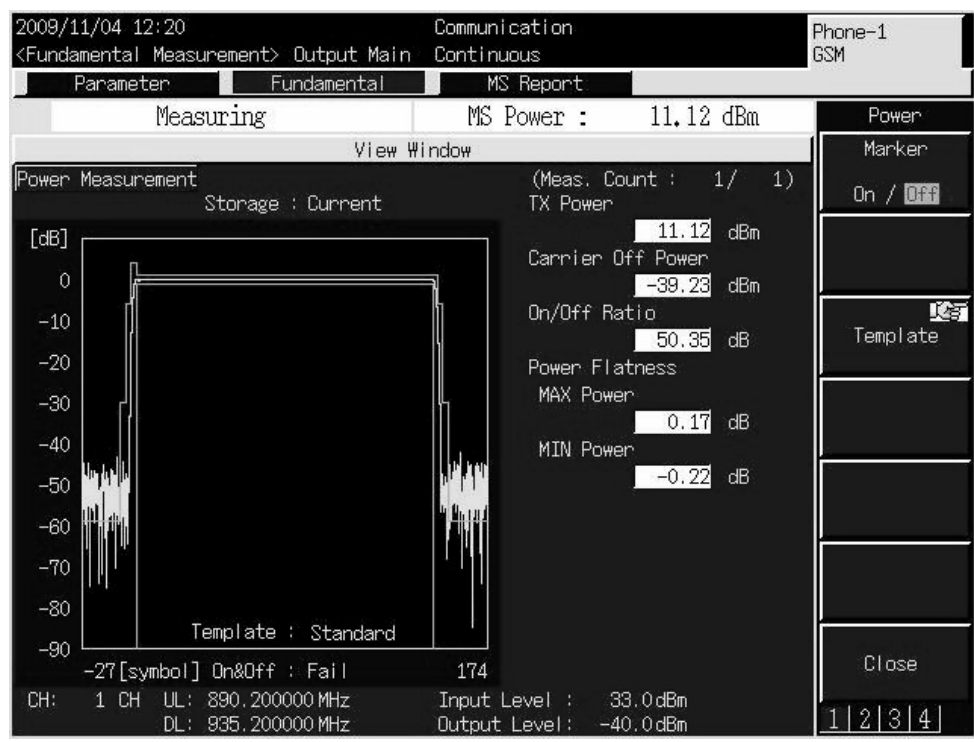

Figure 6. Power measurement

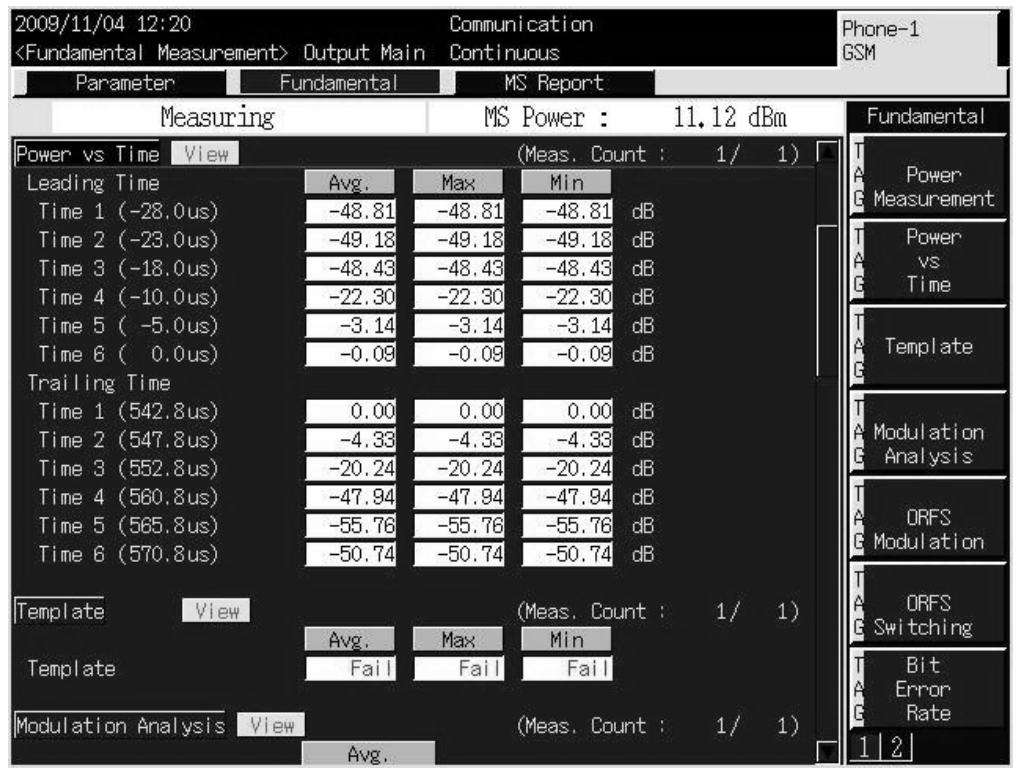

Figure 7. Measurement Power vs time

In this scenery, it does not appeared errors, because the communication is efficient.

Minimum reception POWER MEASUREMENTS IN VOICE CALLS

The second part of the test consists in decreasing the power level of the base station detecting errors in the transmission; the power in which the Nokia equipment established the communication without errors is $-86 \mathrm{dBm}$.

The same test was applied to the mobile iPhone, the power where error came up were $-80 \mathrm{dBm}$. The figures 8-9 show the results got for this mobile. 


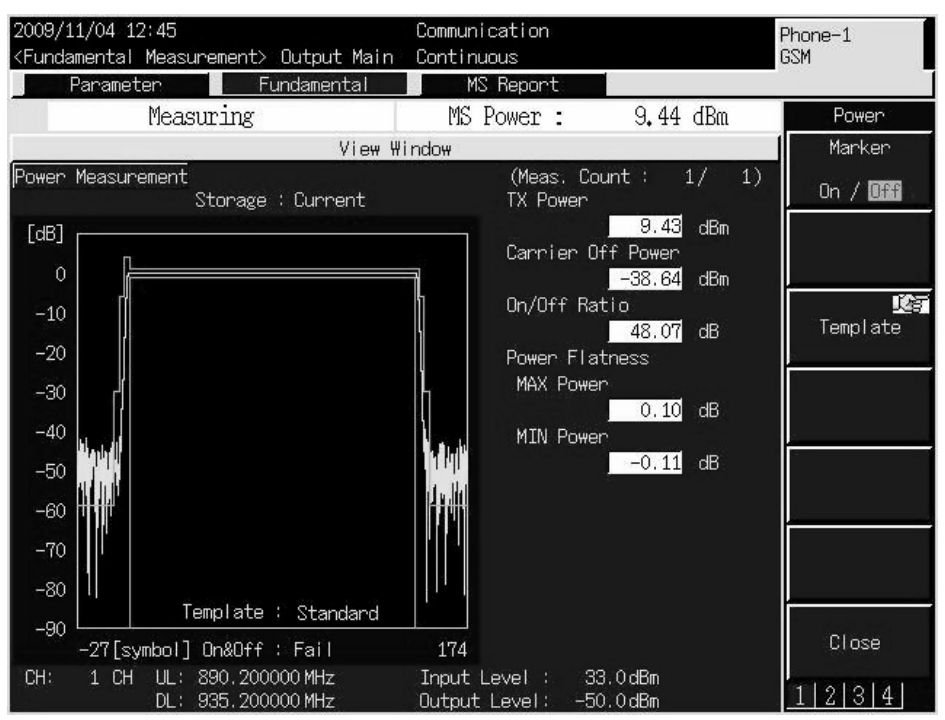

Figure 8. Power measurement

It should be pointed out that Nokia 6120 as iPhone initially satisfy the measurements parameters. As much as the power level is decreased differences between them appear. It should be pointed out that initially the Nokia 6120 as iPhone satisfy the measurements parameters. Once the power level is decreased, we can notice that the Nokia 6120 has better reception sensitivity; the errors in the transmission are detected until $-86 \mathrm{dBm}$, and the minimum power level without errors for the iPhone is $-80 \mathrm{dBm}$. This difference $(6 \mathrm{dBm})$ is a big deal; due to the distance that the Nokia can establish a voice call is greater than iPhone.

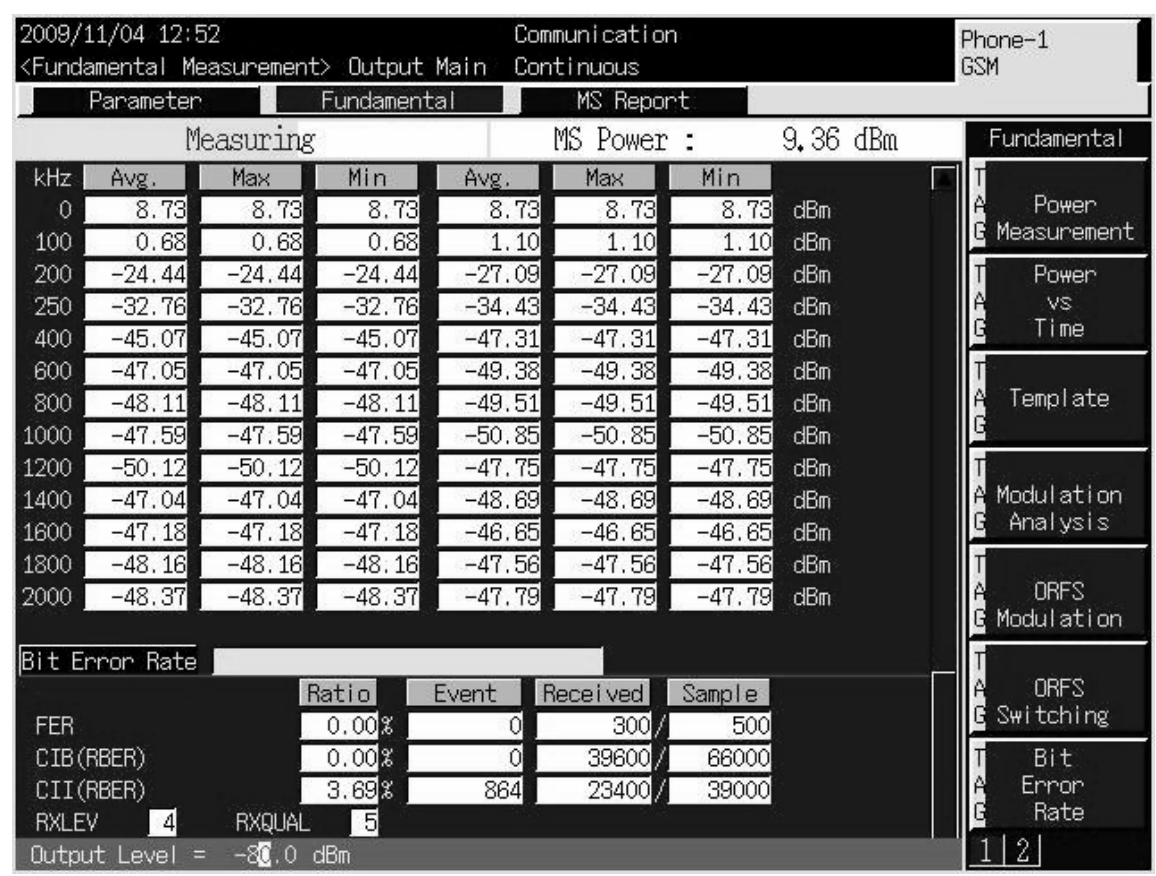


Figure 9. Measurements of BER

\section{Performance Parameters in Mobile Terminals in Edge and WCDMA System}

The mobile terminal is a critical issue inside of a Cellular Communication System, due to its characteristics of transmission and reception; they defined the quality in the data and voice transmission. In this work, some experiments of the most important parameters in Mobile terminal were carried out. In order to make the experimental analysis for EDGE and WCDMA, the following scenario was deployed $[2,3]$.

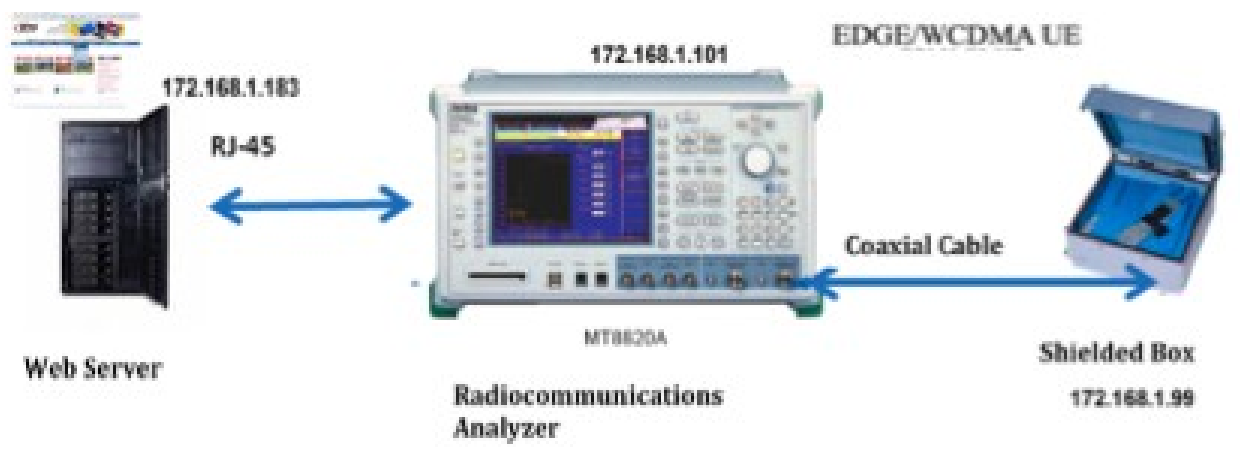

Figure 10. General Test Scenario for EDGE and WCDMA Measurements

\section{Experimental scenario}

The elements that are necessary to figure out the experimental scenario are:

- MT8820A Radiocommunications Analyzer, it has a range of frequencies between 30 $\mathrm{MHz}$ to $2.7 \mathrm{GHz}$, the range result enough to get the experimental measures into the cell phones. This equipment allowed getting measurements of Transmission/Reception in technologies like: W-CDMA/HSDPA, GSM/GPRS/EGPRS, cdma2000, 1x (IS-2000), cdma2000 1xEV-DO, PDC and PHS/ADVANCED [2,3].

- A Shielded Box is used to avoid interferences from the surrounding Base Stations. Such device works between 800 and $2500 \mathrm{MHz}$, and gives $60 \mathrm{~dB}$ of isolation $[2,3]$.

- Coaxial cable is used to connect the MT8820A to the Shielded box. Type-N connectors are used

- Two mobile terminals that operate in EDGE and WCDMA technology. (iPhone 3G and Nokia 6210).

- A USIM card is installed for the test. It was necessary to register the USIM with the Radiocommunications analyzer.

- A data Server (http) is connected into the same network segment that the MT8820A, and the connectivity between them is verified.

\section{EDGE Measurements}

To implement the test scenario shown above (Figure 10) Radio analyzer was configured with the parameters shown in Figure 11. We can observe that EGPRS operation is selected, the power level of base station power is $-50 \mathrm{dBm}$, the power level of the Mobile Terminal is $25 \mathrm{dBm}$, the 
frequencies of the uplink and downlink are $890.2 \mathrm{MHz}$ and $935.2 \mathrm{MHz}$ respectively, and sets the power control, the power level received from the base station by the mobile is $7.89 \mathrm{dBm}$

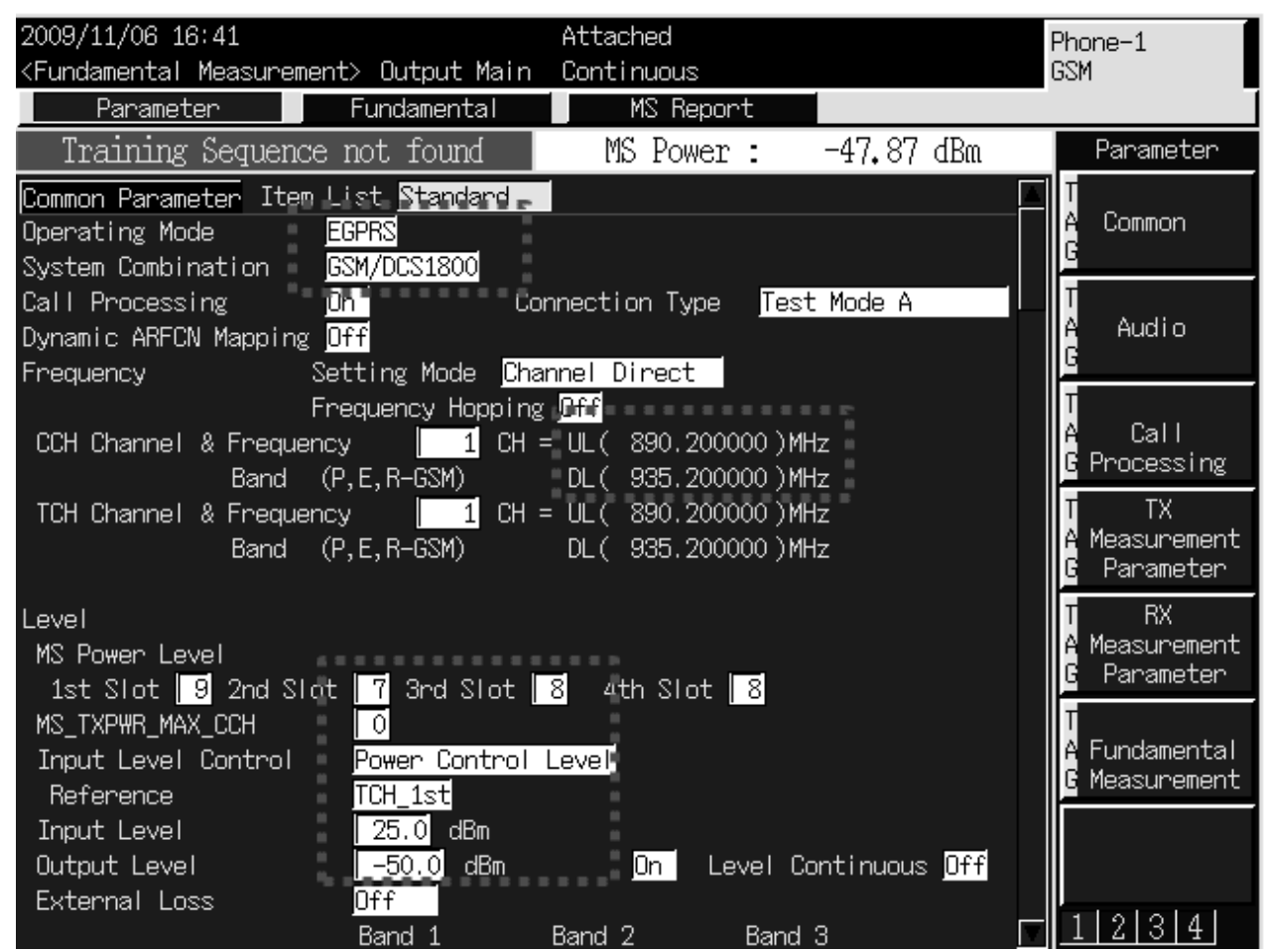

Figure 11. Parameter configurations for EDGE Technology

In addition to GMSK, 8PSK is used by EDGE for higher modulation/coding schemes, this means that for every phase shift we can get a 3-bit word, resulting in three times the rate of transmission of GSM. EDGE uses an adaptive algorithm that adapts the transmission rate (scheme of modulation/coding) according to the radio link quality. This technology theoretically can carry data rates of up to $236.8 \mathrm{Kbps}$ in 4 time slots in packet mode. EDGE is 4 times more efficient than GPRS. GPRS uses four coding schemes (CS-1 to 4), while EDGE uses nine schemes of modulation / coding (MCS-1 to 9) [4].

\section{BLER Test Mode}

BLER test mode permits to estimate the error rate calculated by the mobile terminal and reported to the base station, it is recalled that BLER mode only transmitted GMSK modulation. Just as in GPRS, the BLER is the ratio between the numbers of blocks without acknowledge (errors in the transmission of the block) to the number of blocks successfully confirmed (block successfully transmitted) by the mobile terminal. Figure 12, shows the EDGE settings that are used. 


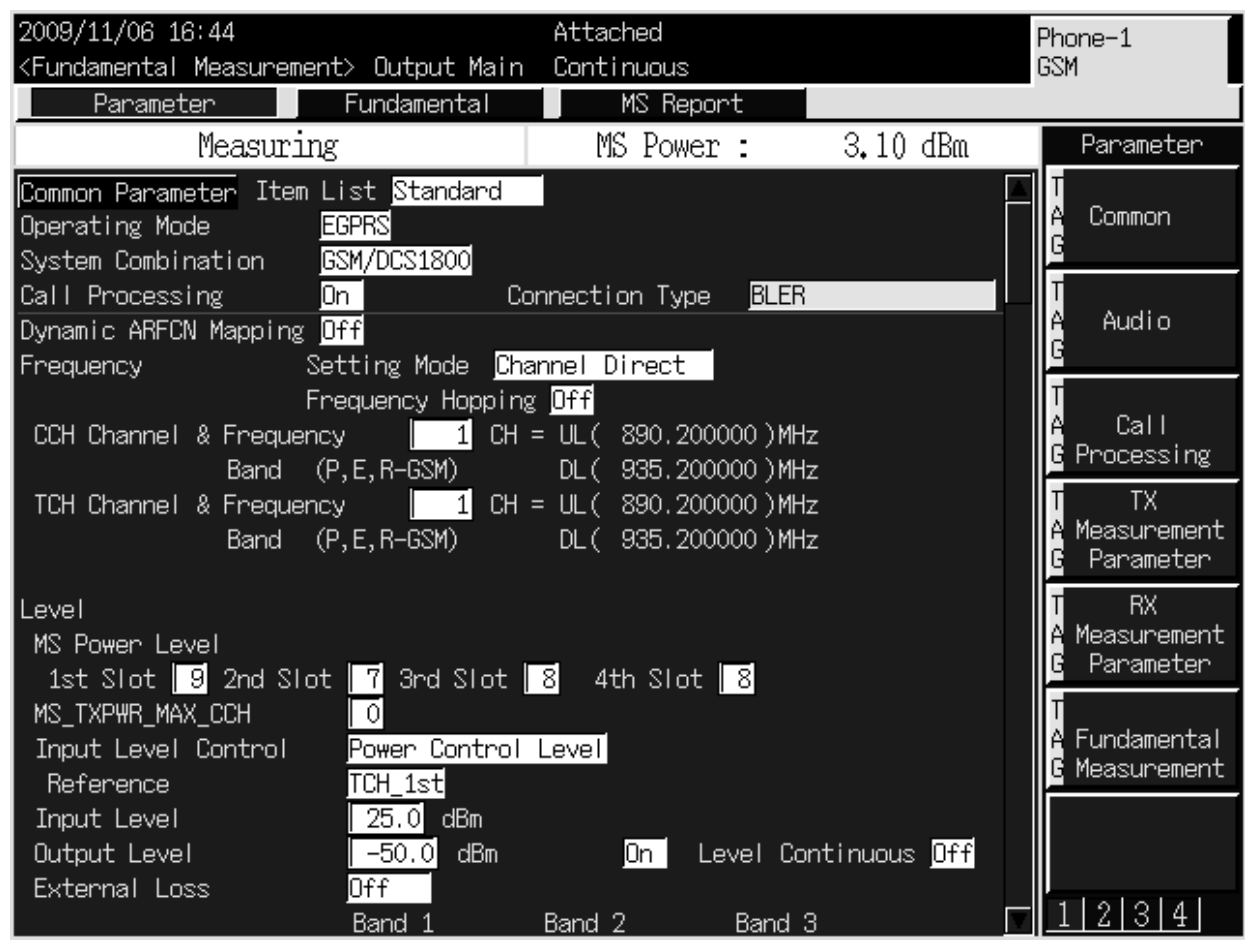

Figure 12. BLER TEST Mode for EDGE

For the BLER mode, two schemes of modulation/coding were selected: MCS-4, which has a GMSK modulation, and MCS-9, which uses 8PSK. The Mobile handsets (Nokia and iPhone) were connected to the MT8820A and its power level was continuously decreasing until we can observe errors in the screen. The results are shown in Table 1

Table 1. BLER Test results.

\begin{tabular}{ccc}
\hline $\begin{array}{c}\text { Terminal } \\
\text { Móvil }\end{array}$ & $\begin{array}{c}\text { Scheme of } \\
\text { modulation/coding }\end{array}$ & $\begin{array}{c}\text { Minimum power } \\
\text { level without } \\
\text { errors }\end{array}$ \\
\hline Nokia 6120 & MCS-4 & $-79 \mathrm{dBm}$ \\
Nokia 6120 & MCS-9 & $-69 \mathrm{dBm}$ \\
iPhone & MCS-4 & $-72 \mathrm{dBm}$ \\
iPhone & MCS-9 & $-64 \mathrm{dBm}$ \\
\hline
\end{tabular}

As we can see (the results in Table 1), MCS-9 is used to transmit a greater number of bits, but requires more power to carry out their work, while with MCS-4 needs lower power level and continues transmitting without errors. Figures 13 and 14 show the power level measurements. 


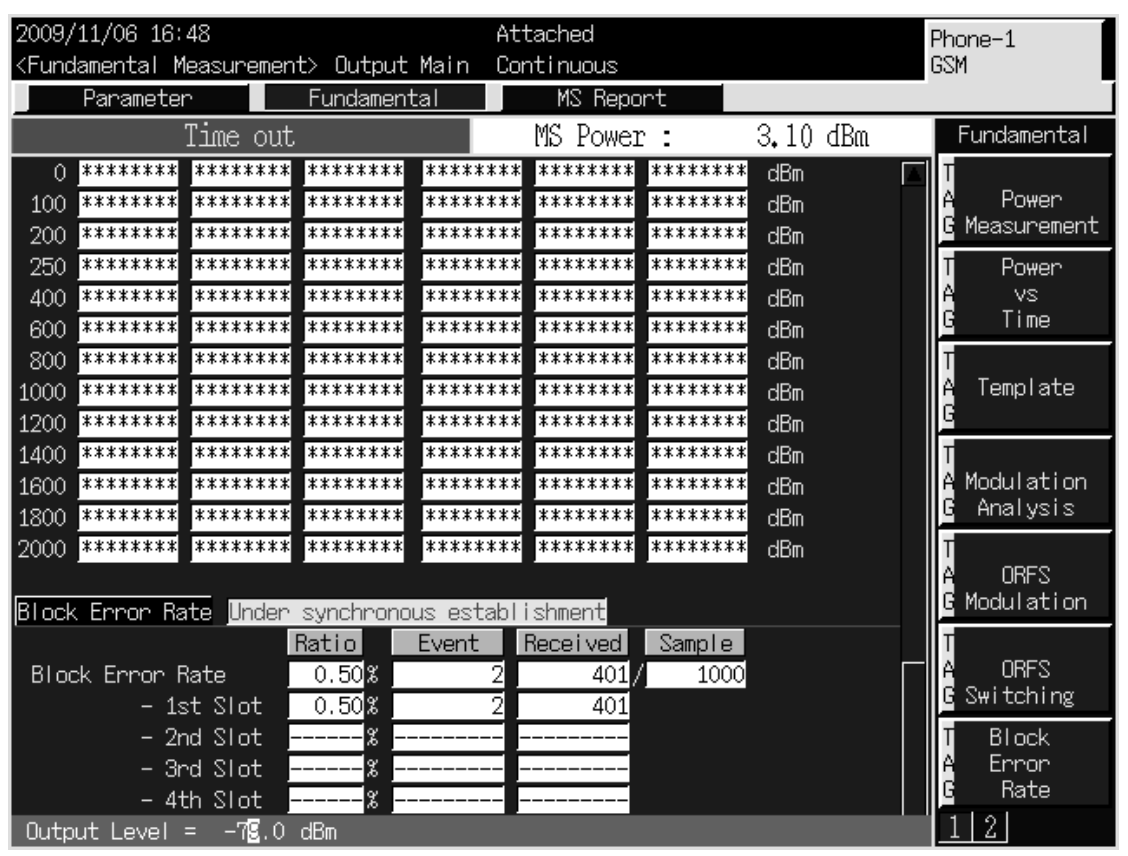

Figure 13. BLER Test with MSC-4

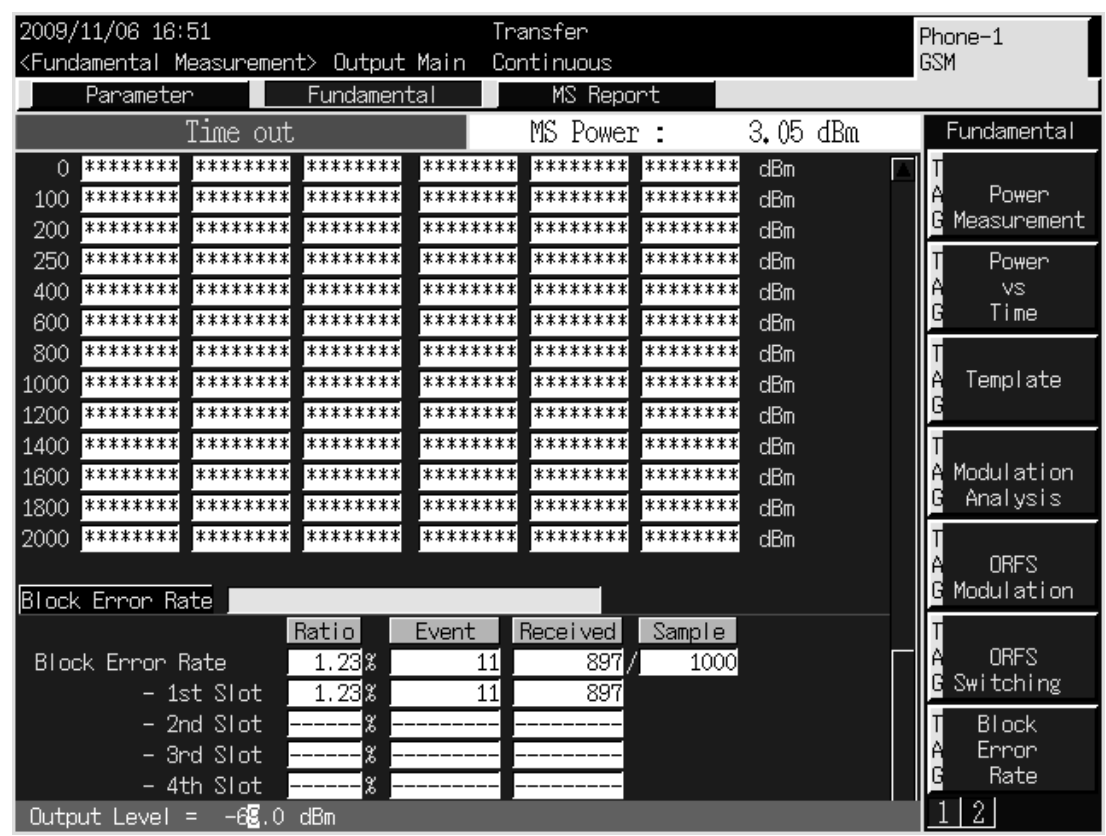

Figure 14. BLER test with MSC-9

Since EDGE can assign multiple time slots for data transmission, 4 slots for the uplink were configured (UL) and 1 slot for downstream (DL) with a MCS-9 scheme, but the power level to which errors were observed is $-69 \mathrm{dBm}$ (like the previous measurement). In the same way, when we configured a MSC-4 scheme with 4DL, 1UL; the power level to which errors occur is $-79 \mathrm{dBm}$ (like to that found with a single uplink and downlink) 


\section{WCDMA Measurements}

For the WCDMA measurements, the MT8820A were configured as follows (Figure 15);

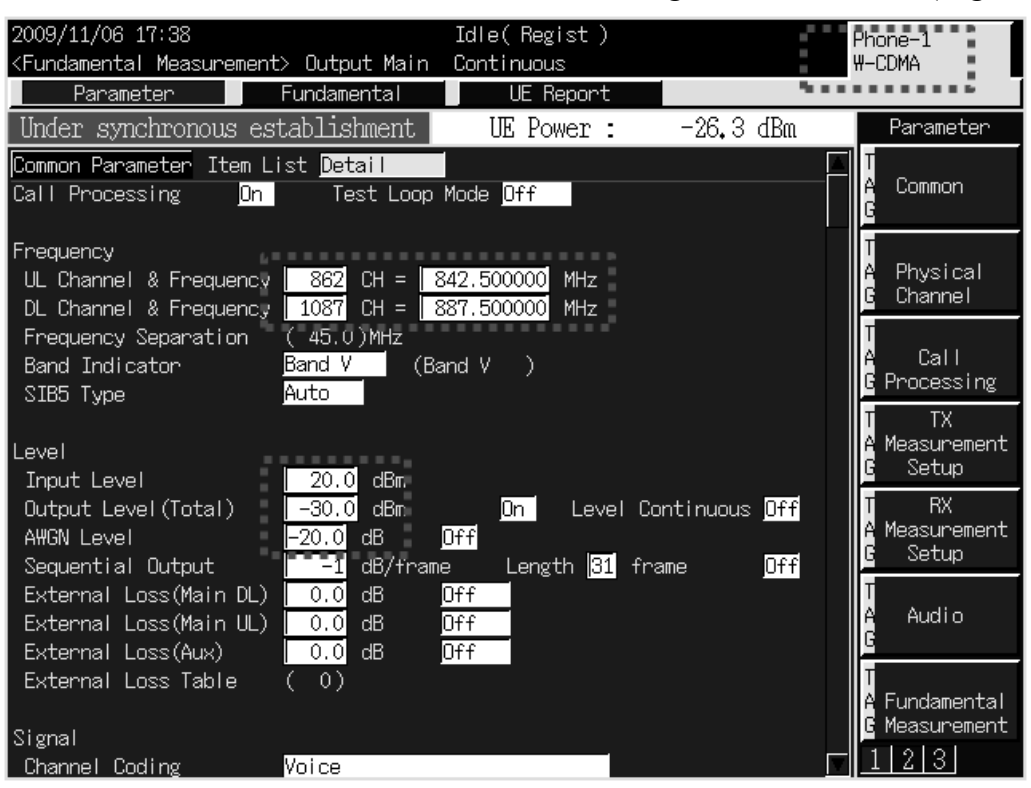

Figure 15. MT8820A configuration for WCDMA

For the testing of WCDMA, the band V of the UMTS channels is selected [5], corresponding to Mexico; the channels $1087(887.5 \mathrm{MHz})$ and $862(842.5 \mathrm{MHz})$ are used respectively for downlink and uplink. We used a power level of $-30 \mathrm{dBm}$ for the transmission of MT8820A

\section{BER/BLER Measurements}

As in EDGE, BER test mode is configured in the MT8820A and it proceeded to decrease the power level until we observe errors in the Analyzer. The Table 2 and Figure 16 show the results of the two terminals under evaluation.

Table 2. BER/BLER results.

\begin{tabular}{ccc}
\hline User Equipment & $\begin{array}{c}\text { Power level } \\
\text { without errors }\end{array}$ & $\begin{array}{c}\text { Power level } \\
\text { with errors }\end{array}$ \\
\hline $\begin{array}{c}\text { Nokia 6120 } \\
\text { iPhone }\end{array}$ & $-80 \mathrm{dBm}$ & $-82 \mathrm{dBm}$ \\
& $-74 \mathrm{dBm}$ & $-75 \mathrm{dBm}$ \\
\hline
\end{tabular}




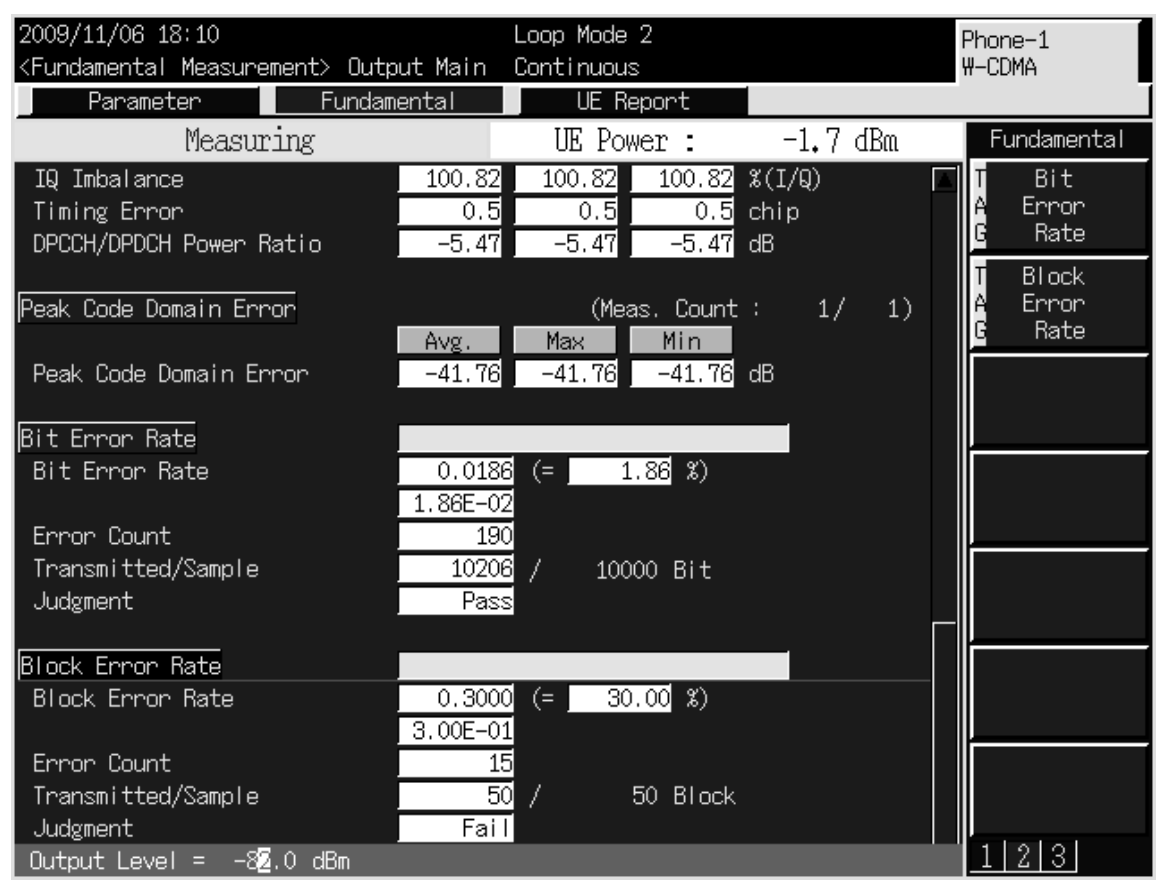

Figure 16. BER/BLER results for Nokia 6120

\section{External Data Measurements}

As with GPRS and EDGE, WCDMA technology allows data transmission tests from an external server to the mobile terminal via PPP and IP connections. Radio Analyzer (MT8820A) enables communication between a server and a client using the air interface of the mobile terminal. The scenario is shown in Figure 14.

For this test, the following parameters were configured in the MT8820A [4].

- The IP Default Gateway field is set to 0.0.0.0

- Call Processing parameter must be enabled,

- The Test Loop Mode must be disabled and

- The Channel coding must be Packet, to indicate that we establish a data session.

- The IP addressing is shown in Figure 10.

Subsequently, we introduce the mobile terminal within the shielded box and the Analyzer proceeds to Register the equipment under test, and once this is done, the Communication mode is set indicating that the equipments are linked and data transfer can take place. In this case, due to the characteristics of the measuring equipment, we can only test the downlink 384Kbps theoretical speed [6,7]. The power level configuration of the base station and mobile terminal was carried out according to what is specified in Figure 15. Once that is done all the configuration and connectivity testing, we proceed to the download of information from a local web site to test the transmission rates using different coding schemes.

The user equipment downloaded a file (200KB) from a local web server (Figure 10). Table 3, shows the results of the test. 
Table 3. Data rate for WCDMA

\begin{tabular}{ccccc}
\hline $\begin{array}{c}\text { Tamaño de } \\
\text { archivo } \\
\text { (KB) }\end{array}$ & $\begin{array}{c}\text { Tamaño de } \\
\text { Archivo (bits) }\end{array}$ & $\begin{array}{c}\text { Tiempo de } \\
\text { Transferencia } \\
\text { (seg) }\end{array}$ & $\begin{array}{c}\text { Tasa de } \\
\text { Transmisión } \\
\text { Medida (Kbps) }\end{array}$ & $\begin{array}{c}\text { Tasa de } \\
\text { Transmisión } \\
\text { Teórica (Kbps) }\end{array}$ \\
\hline $\mathbf{2 0 0}$ & 1600000 & 5.67 & 292.68 & 384 \\
\hline
\end{tabular}

As we can see, there is a difference between the data rate measured and data rate theoretical provided by the WCDMA downlink. As with GPRS, the information added by the IP transmission, as well the WCDMA air interface caused an increase in the amount of information transmitted in the communication, which influence the transmission rate measured.

\section{Conclusions}

This paper shows the experimental results done to the two mobile terminals in GSM and GPRS systems. It was verified in the beginning that the terminals have the required specifications. Later they were measure in which the terminals started to present errors, and it results indicators of the ratio of the cells. For the transmission of data, it was found the power limits changing the modulation schemes. The limit power is an important data for designing and planning of a radio network. This task established the tests for a deep analysis from the point of view of coverage in capacity of radio. In future tasks analysis of capacity in transmission will be presented depending on the distance, slots and modulation schemes. It should be remarked that this kind of research is important, due to their results has a great impact at different levels; like that planning, research, manufacturing, scientific and academic areas.

In this paper is shown the experimental results done to the two mobile terminals in EDGE and WCDMA systems. Measurements were based on BER/BLER and data transmission. In first place, we were able to find out the minimum power level, which user equipments can transmit without errors. This fact is too important for planning the cell dimension for EDGE and WCDMA networks. In the other hand, as shown in the table 14, the measured results differ from what shows the specification. This difference can be attributed to different issues like: The headers and trailers added by each of the layers in the IP segment (TCP, IP, Ethernet, Physical Layer) as well as the wireless segment of EDGE and WCDMA, different processing capabilities of mobile terminals, Network Congestion.

The minimum receiving power level is an important data for designing and planning of a radio network. This task established the tests for a deep analysis from the point of view of coverage in capacity of radio. In future tasks analysis of capacity in transmission will be presented depending on the distance, slots and modulation schemes. It should be remarked that this kind of research is important, due to their results has a great impact at different levels; like that planning, research, manufacturing, scientific and academic areas. 


\section{REFERENCES}

[1] Application Note No 11410-00378 Rev. B, "Practical Tips on WCDMA Measurements", Anritsu, 2009. Available in: http://www.us.anritsu.com

[2] Christophe Chevallier, Christopher Brunner, Andrea Garavaglia, Kevin P. Murray, Kenneth R. Baker, WCDMA Deployment Handbook Planning and Optimization Aspects, John Wiley \& Sons, 2006

[3] Harri Holma and Antti Toskala, WCDMA for UMTS- Radio Access for Third Generation Mobile Communications, John Wiley \& Sons, 2004

[4] Keiji Tachikawa, WCDMA Mobile Communications System, John Wiley \& Sons, 2002

[5] Practical Tips on WCDMA Measurements, Application Note No. 11410-00378, Rev. B Printed in United States 2008-09

[6] Sistemas Electrónicos de Comunicaciones, Roy Blake, Thomson, 2th Edition 2004

[7] WCDMA for UMTS-HSPA Evolution and LTE, Harri Holma and Antti Toskala, Wiley, 4th edition 2007 